\title{
Saponin constituents of Achyranthes root
}

\author{
Fumiyuki Kiuchi $^{1}$ (1)
}

Received: 8 October 2021 / Accepted: 30 November 2021 / Published online: 4 January 2022

(c) The Author(s) 2021

\begin{abstract}
Achyranthes root is a crude drug used as diuretic, tonic and remedy for blood stasis. Characteristic oleanolic acid saponins with a dicarboxylic acid moiety have been isolated as one of the representative constituents of this crude drug. This review focuses on the triterpene saponin constituents, especially those with a characteristic dicarboxylic acid moiety, of A. bidentata and A. fauriei. Several groups isolated the saponins and different names were given to one compound in some cases. The names of the compounds are sorted out and the stereochemistry of the dicarboxylic acid moieties are summarized. HPLC analysis of the composition of the saponin constituents and the effect of processing and extraction conditions on the composition are reviewed. Biological activities of the saponin constituents are also summarized.
\end{abstract}

Keywords Achyranthes root $\cdot$ Achyranthes bidentata $\cdot$ Achyranthes fauriei $\cdot$ Oleanane saponin $\cdot$ Achyranthoside . Betavulgaroside

\section{Introduction}

Achyranthes root is a crude drug used as diuretic, tonic, and remedy for blood stasis. In the Japanese Pharmacopoeia, the origin of this crude drug is defined as the root of Achyranthes fauriei Leveille et Vaniot or A. bidentata Blume (Amaranthaceae) [1]. However, in the Plants of the World Online [2], A. fauriei is one of the 19 synonyms of A. bidentata. A comprehensive review, covering literatures published up to 2016, of the two medicinal species of Achyranthes, A. bidentata and $A$. aspera, on the traditional uses, phytochemistry, and pharmacological activities has been published [3]. Phytoecdysones, triterpene saponins, polysaccharides, and polypeptides are the four major classes of the bioactive constituents of Achyranthes root. Phytoecdysones are the first bioactive constituents isolated from A. fauriei [4, 5], and the triterpene saponins have characteristic dicarboxylic acid substituents. In recent years, more attention has been paid to the high molecular weight constituents of Achyranthes root, i.e. polypeptides and polysaccharides, in relation to the biological activities [3]. This review focuses on the triterpene

Fumiyuki Kiuchi

kiuchi-fm84@keio.jp

1 Faculty of Pharmacy, Keio University, 1-5-30 Shibakoen, Minato-ku, Tokyo 105-8512, Japan saponin constituents, especially those with a characteristic dicarboxylic acid moiety, of A. bidentata and A. fauriei.

\section{Isolation and characterization of saponin constituents}

Achyranthes root contains characteristic oleanane saponins with dicarboxylic acid substituents. These saponins have been isolated by different groups and, in some cases, different names were given to one compound. The structures and names of the saponins are summarized in Fig. 1 and Table 1. In the following text, original names described in the literature are used and, if necessary, the names listed in the first column of Table 1 are followed in parenthesis. The ${ }^{13} \mathrm{C}$ NMR data of the saponins with dicarboxylic acid moiety reported in the literatures are summarized in Table S1 (Supplementary Material).

Four triterpene saponins were first isolated and characterized from the $\mathrm{BuOH}$ soluble fraction of the $\mathrm{MeOH}$ extract of Achyranthes root (the root of A. fauriei) by Ida et al. [6]. They treated the $\mathrm{BuOH}$ soluble fraction with diazomethane and isolated methyl esters of chikusetsusaponins (CSs) IVa (1) and V (2), pseudoginsenoside $\mathrm{RT}_{1}(\mathbf{3})$, and 28-desglucosylchikusetsusaponin V (4). These compounds are oleanane saponins with glucuronic acid at C-3. From the same source, Ida et al. [7-10] also isolated oleanane saponins, 


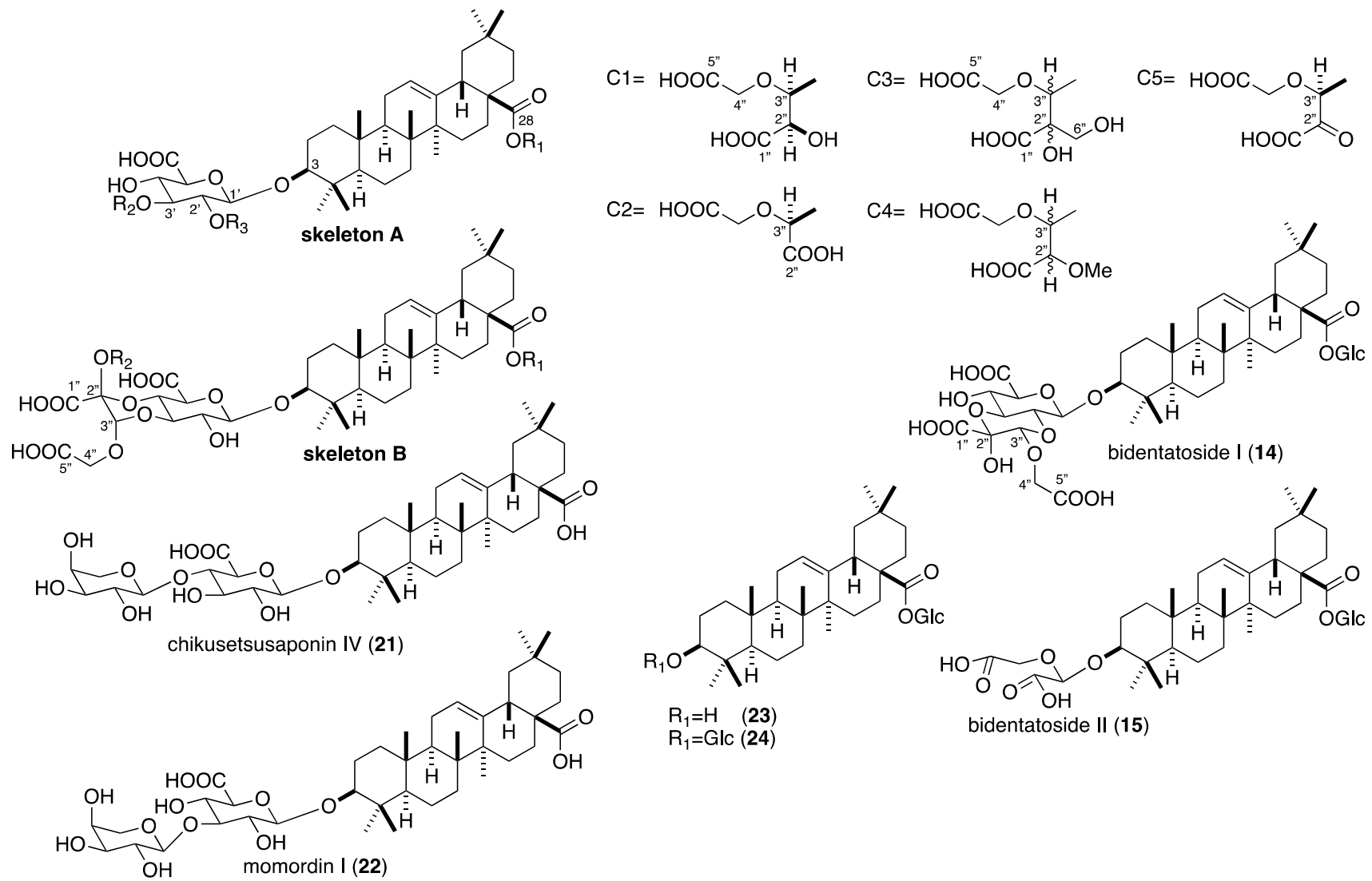

Fig. 1 Structures of the saponins isolated from Achyranthes root and related compounds. C-3" of the dicarboxylic acid moieties $\mathrm{C} 1-\mathrm{C} 5$ attaches to C-3' of the glucuronic acid moiety of skeleton A through an ether linkage. C-2" of C5 also forms a hemiacetal linkage to C-4' of the glucuronic acid moiety to form skeleton B

G, respectively. Similar oleanane saponins with a characteristic dicarboxylic acid moiety were also isolated from the $\mathrm{BuOH}$ soluble fraction of the $\mathrm{MeOH}$ extract of $A$. bidentata by a French group and named bidentatosides (BDSs) I (14) and II (15) [17, 18]. BDS I (14) has a dioxane ring similar to that of AS B (6). However, the positions of the acetal linkages are not $\mathrm{C}-3^{\prime}$ and $\mathrm{C}-4^{\prime}$ but $\mathrm{C}-2^{\prime}$ and $\mathrm{C}-3^{\prime}$ of the glucuronic acid moiety [17]. BDS II (15) lacks the glucuronic acid moiety, and the dicarboxylic acid attaches directly to the C-3 of oleanolic acid. They also isolated three oleanolic acid saponins without dicarboxylic acid moiety [oleanolic acid-28- $O$ - $\beta$-D-glucopyranoside (23), CS V (2), and 3-O- $\beta$ D-glucopyranosyl-oleanolic acid-28- $O$ - $\beta$-D-glucopyranoside (24)] from the roots of $A$. bidentata [19]. Momordin Ib (16) was also isolated from the $\mathrm{MeOH}$ extract of $A$. bidentata together with ASs B (6) and E (9), and CSs IVa (1) and V (2), and their methyl esters [20]. Methyl ester of 28-desglucosylachyranthoside D (=AS G, 11) was also isolated from the $\mathrm{MeOH}$ extract of $A$. bidentata [21], and ASs C (7) and D (8) together with CS IV (21), ginsenoside Ro (=CS V, 2), and zingibroside $R_{1}$ (=28-desglucosyl CS V, 4) were isolated from the $\mathrm{BuOH}$ soluble fraction of the $70 \% \mathrm{EtOH}$ 
Table 1 Summary of the saponins isolated from Achyranthes root and related compounds

\begin{tabular}{|c|c|c|c|c|c|c|}
\hline Compound & Other names & Skeleton & $\mathrm{R}_{1}$ & $\mathrm{R}_{2}$ & $\mathrm{R}_{3}$ & References \\
\hline Chikusetsusaponin IVa (1) & & A & Glc & $\mathrm{H}$ & $\mathrm{H}$ & {$[6]$} \\
\hline Chikusetsusaponin V (2) & Ginsenoside Ro & A & Glc & $\mathrm{H}$ & Glc & {$[6,19,22]$} \\
\hline Pseudoginsenoside $\mathrm{RT}_{1}(\mathbf{3})$ & & A & Glc & $\mathrm{H}$ & Xyl & {$[6]$} \\
\hline $\begin{array}{l}\text { 28-Desglucosyl- } \\
\text { Chikusetsusaponin V (4) }\end{array}$ & Zingibroside $\mathrm{R}_{1}$ & A & $\mathrm{H}$ & $\mathrm{H}$ & Glc & {$[6,22]$} \\
\hline Achyranthoside A (5) & & $\mathrm{B}$ & Glc & $\mathrm{Me}$ & & [7] \\
\hline Achyranthoside B (6) & $\begin{array}{l}\text { Betavulgaroside I } \\
\text { Achyranthoside III }\end{array}$ & $\mathrm{B}$ & Glc & $\mathrm{H}$ & & {$[7,11,16,20,24]$} \\
\hline Achyranthoside C (7) & Betavulgaroside III & A & Glc & $\mathrm{C} 1$ & $\mathrm{H}$ & {$[8,10,11,22,23,25]$} \\
\hline Achyranthoside D (8) & $\begin{array}{l}\text { Betavulgaroside V } \\
\text { Achyranthoside I }\end{array}$ & A & Glc & $\mathrm{C} 1$ & Glc & {$[8,10,11,15,22,24]$} \\
\hline Achyranthoside E (9) & Spinacoside C & A & Glc & $\mathrm{C} 2$ & $\mathrm{H}$ & {$[9,14,20,23,25]$} \\
\hline Achyranthoside F (10) & & A & Glc & $\mathrm{C} 3$ & $\mathrm{H}$ & [9] \\
\hline Achyranthoside G (11) & Achyranthoside IV & A & $\mathrm{H}$ & $\mathrm{C} 1$ & Glc & {$[10,16,21,25]$} \\
\hline Achyranthoside H (12) & & A & Glc & $\mathrm{C} 4$ & $\mathrm{H}$ & {$[10]$} \\
\hline Betavulgaroside IV (13) & Achyranthoside II & A & $\mathrm{H}$ & $\mathrm{C} 1$ & $\mathrm{H}$ & {$[11,15,25]$} \\
\hline Bidentatoside I (14) & & & & & & {$[17]$} \\
\hline Bidentatoside II (15) & & & & & & {$[18]$} \\
\hline Momordin Ib (16) & & A & $\mathrm{H}$ & $\mathrm{H}$ & $\mathrm{H}$ & {$[20]$} \\
\hline Sulfachyranthoside B (17) & & $\mathrm{B}$ & Glc-4- $\mathrm{O}-\mathrm{SO}_{3} \mathrm{H}$ & $\mathrm{H}$ & & {$[24]$} \\
\hline Sulfachyranthoside D (18) & & A & Glc-4- $O-\mathrm{SO}_{3} \mathrm{H}$ & $\mathrm{C} 1$ & Glc & {$[24]$} \\
\hline Betavulgaroside II (19) & & $\mathrm{B}$ & $\mathrm{H}$ & $\mathrm{H}$ & & {$[11,25]$} \\
\hline Spinacoside D (20) & & A & $\mathrm{H}$ & $\mathrm{C} 2$ & $\mathrm{H}$ & {$[14]$} \\
\hline Momordin I (22) & & A & $\mathrm{H}$ & Ara & $\mathrm{H}$ & {$[28]$} \\
\hline
\end{tabular}

A, B, C1-C5: see Fig. 1

Ara $\alpha$-L-arabinopyranosyl, Glc $\beta$-D-glucopyranosyl, Glc-4- $O-\mathrm{SO}_{3} \mathrm{H}$ 4-O-sulfooxy- $\beta$-D-glucopyranosyl, $X y l \quad \beta$-D-xylopyranosyl

extract of A. bidentata [22]. Methyl and butyl esters of ASs $\mathrm{C}$ (7) and $\mathrm{E}(9)$ were also isolated from the $\mathrm{BuOH}$ soluble fraction of the $\mathrm{MeOH}$ extract of A. bidentata [23]. Two achyranthoside derivatives, named sulfachyranthosides (SASs) B (17) and D (18), which were sulfated at the C-4'" of the glucose moiety at C-28 of oleanolic acid, together with AS $\mathrm{D}(\mathbf{8})$ were isolated from a water extract of Achyranthes root [24], and ASs C (7), E (9), G (11), and BVSs II (19) and IV (13) were identified from the water extract of Achyranthes root by the same group [25]. The plant sources of achyranthoside derivatives are limited. The other plants include Basella rubra (Basellaceae) for BVS I (= AS B, 6) and SS C (=AS E, 9) [26], and Pisonia umbellifera (Nyctaginaceae) for AS E (9) and SS D (20) [27].

\section{Stereochemistry of the dicarboxylic acid moiety}

Five types of the dicarboxylic acid moiety (C1-C5 in Fig. 1) have been reported for the saponins isolated from Achyranthes root. Dicarboxylic acid C5 forms the 1,4-dioxane ring of the skeleton B and BDS I (14) with the glucuronic acid moiety (Fig. 1). The stereochemistry of AS B (6) was established by an X-ray crystallographic analysis of its 28 -desglucosyl derivative as shown in Fig. 1 [7]. Yoshikawa et al. established the absolute stereostructures of BVSs III (= AS $\mathrm{C}, \mathbf{7})$ and IV (13) by chemical correlations to momordin I (22) (Fig. 2). The $\alpha$-L-arabinosyl moiety of 22 [28] was converted to $\alpha$-L-ribosyl derivative (X1) whose cis diol was then oxidized to give compound $\mathbf{X} 2$, which was identical with the compound derived from BVS IV (13) $[29,30]$. Thus, the stereochemistry of the dicarboxylic acid moiety $\mathrm{C} 1$ was determined to be $\left[2^{\prime \prime} R, 3^{\prime \prime} S\right]$ as shown in Fig. 1. They also oxidized the hydroxy group of $\mathrm{C} 1$ of the derivative $\mathbf{X} \mathbf{3}$ to yield $\mathbf{X 4}$, which was identical with the compound obtained by a treatment of BVS II (19) with diazomethane [30]. This established the stereochemistry of the dicarboxylic acid moiety $\mathrm{C} 5$ and the stereochemistry of the dioxane ring, which was in agreement with that of AS B (6) established by the X-ray crystallographic analysis [7]. The stereochemistry of the dicarboxylic acid moiety C2 of SSs C (=AS E, 9) and $\mathrm{D}(\mathbf{2 0})$ was also determined by a chemical correlation to 22 (Fig. 2) [14]. The stereochemistry of BVS III (=AS C, 7) was also confirmed by synthesis starting from oleanolic acid, D-glucose and L-arabinose by Zhu et al. [31]. They 


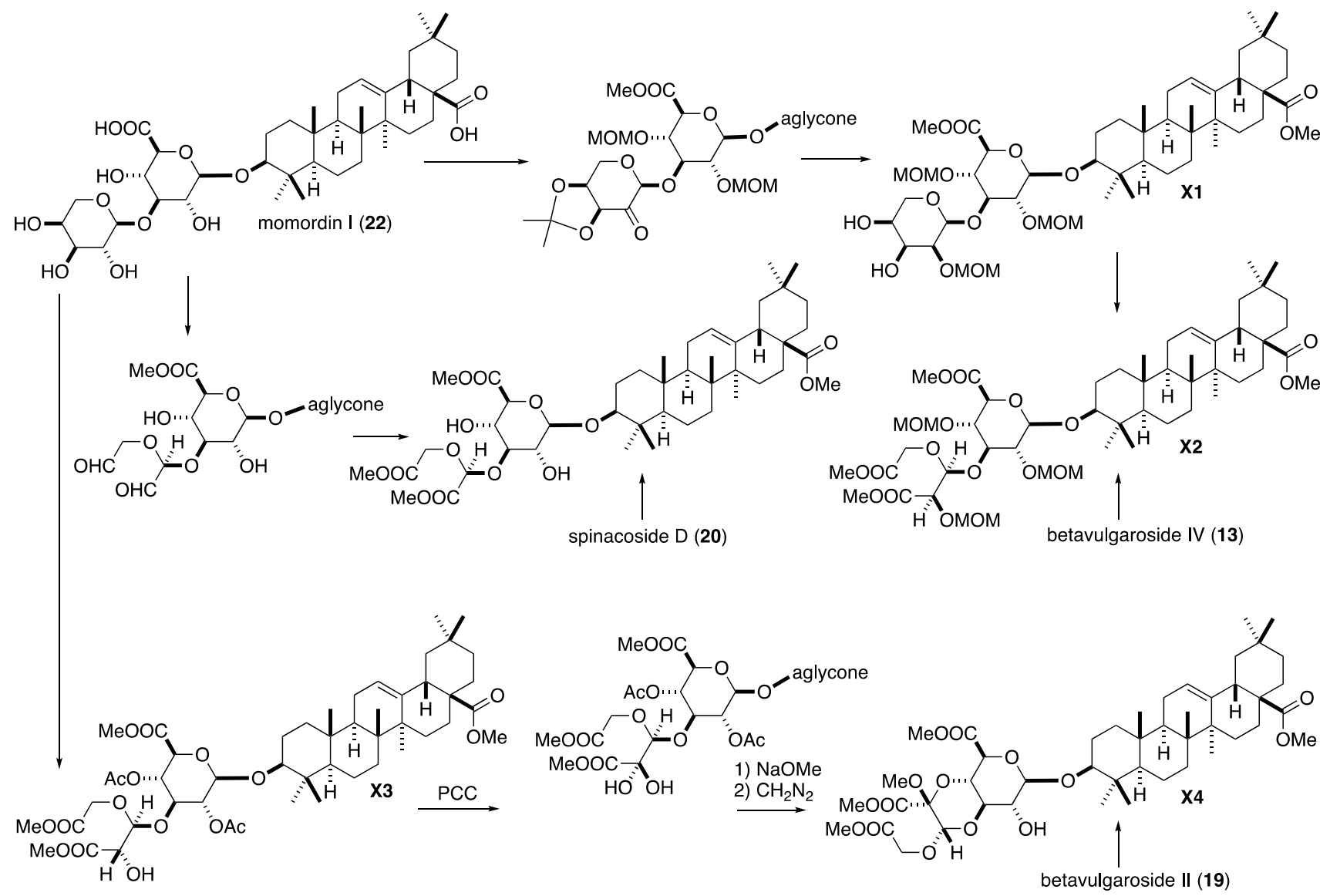

Fig. 2 Chemical correlations of the saponins with momordin I

synthesized BVS III (7) and its C-2" epimer from the same starting materials and showed that the natural $\mathrm{C} 1$ moiety could be derived by oxidative cleavage of 3,4-cis-diol of not the $\alpha$-L-arabinopyranosyl derivative but corresponding $\alpha$-L-ribopyranosyl derivative. Thus, the dicarboxylic acid moiety of ASs B (6), C (= BVS III, 7) and E (=SS C, 9), BVS IV (13), and SS D (20) have a common stereochemistry of [3"S]. As the dicarboxylic acid part of AS B (C5 in Fig. 1) is an oxidized form of the dicarboxylic acid moiety $\mathrm{C} 1$, and the dicarboxylic acid moiety $\mathrm{C} 2$ can be formed oxidative decarboxylation of C5 [32], these results suggested that C1, $\mathrm{C} 2$, and $\mathrm{C} 5$ have a common origin.

As suggested by Yoshikawa et al. [30] and Zhu et al. [31], the dicarboxylic acid moiety can be derived by an oxidative fragmentation of a terminal monosaccharide unit. As described above, the dicarboxylic acid moieties $\mathrm{C} 1, \mathrm{C} 2$ and C5 were correlated to $\alpha$-L-ribopyranosyl group. However, as L-ribose has not been found in nature, $3^{\prime}-\alpha$-L-ribopyranosyl derivatives $\mathbf{2 5}$ are not possible (Fig. 3). Plausible candidates are $\beta$-D-lyxopyranosides $\mathbf{2 6}$, which have the required stereochemistry at C-1 and C-2 corresponding to the C-3" and C-2" of the dicarboxylic acid moiety, respectively. However, such glycosides have not been detected in Achyranthes root. The dicarboxylic acid moiety C3 of achyranthoside $\mathrm{F}$ (10) contains an additional hydroxymethyl group at the 2 "-position of $\mathrm{C} 1$. As the stereochemistry of $\mathrm{C} 3$ has not been determined, a derivative of D-hamamelose (2- $C$-hydroxymethylribose) $\mathbf{2 7}$ is a possible precursor. However, if the stereochemistry of $\mathrm{C} 3$ is the same with that of $\mathrm{C} 1$, the terminal sugar moiety should be its enantiomer. As only a limited number of the saponins with such dicarboxylic acid moiety has been reported, a specific pathway seems to be involved in the biosynthesis of the dicarboxylic acid moieties.

\section{HPLC analysis of saponins}

Li et al. [33] reported an HPLC method to analyze main phytoecdysones and triterpenoids in the root of Achyrantes bidentata. Four ecdysterones, oleanolic acid and six derivatives of oleanolic acid with glucuronic acid moiety at C-3 (1 and its ethyl ester, 2, $\mathbf{4}$ and its butyl ester, 16) were identified using an ODS column and a solvent system containing formic acid. Qualification and quantification method for eight triterpenoids ( $\mathbf{1}$ and its ethyl ester, 2, 4, 16 and its butyl ester, 23, and oleanolic acid) of Achyranthes root by HPLC with 

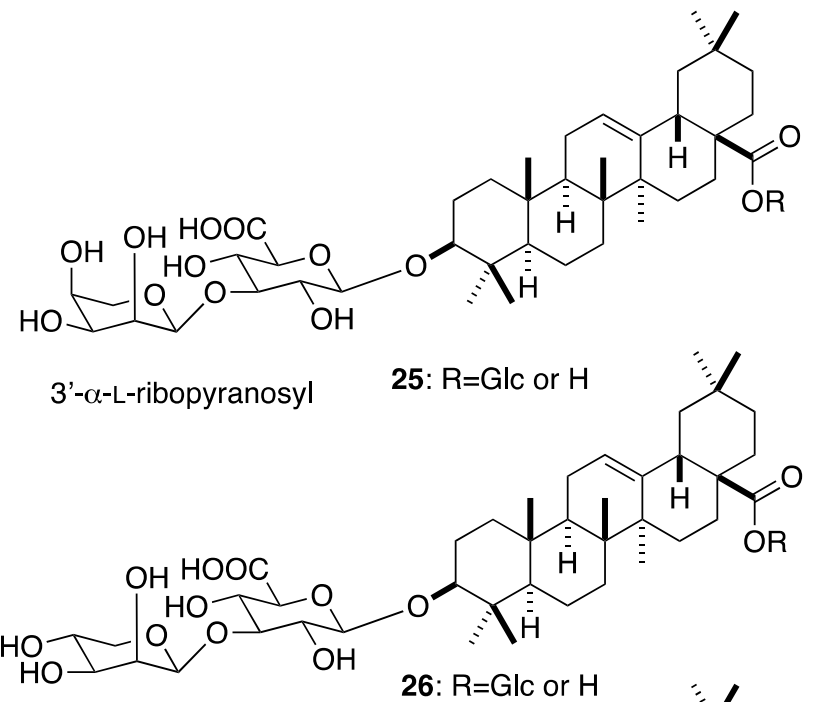

26: $\mathrm{R}=\mathrm{Glc}$ or $\mathrm{H}$

3'- $\beta$-D-lyxopyranosyl

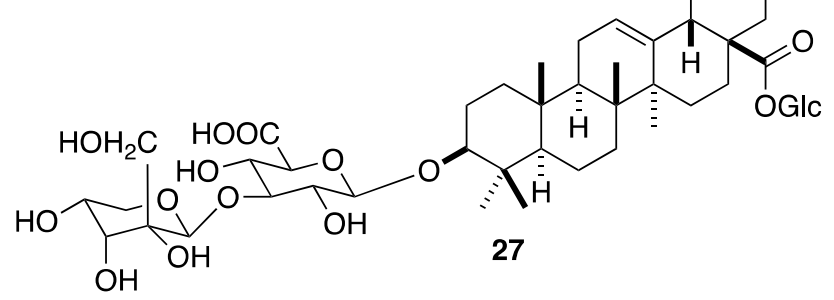

3'- $\beta$-D-hamamelopyranosyl

Fig. 3 Plausible candidates for the precursor of achyranthosides

evaporative light scattering detection and ESI-MS detection was also reported [34]. However, achyranthosides were not detected in these reports.

Li et al. [35] reported an LC-MS/MS method to analyze and characterize saponins in Achyranthes bidentata, using an ODS column with a solvent system containing formic acid. With this method, 22 oleanane-type triterpenoid saponins including ASs C (7) and D (8) were characterized. However, the peaks of achyranthosides showed considerable tailing under this condition. The peak shapes of achyranthosides were greatly improved by the use of cationic ion-pair reagents with reversed-phase column, and an LC-MS based quantification method of achyranthosides using a combination of dihexyl ammonium acetate and phenyl-hexylated silica gel column was reported (Fig. 4) [25]. Under this condition, the retention time of the saponins was dependent on the number and position of acidic groups in each molecule. CSs (1 and 2), which have only one carboxylic acid of glucuronic acid, eluted first. ASs B-E (6-9) with three carboxylic acids eluted next, followed by SASs B and D (17 and 18) which have an additional sulfonic acid on the glucose moiety at C-28 of oleanolic acid. AS G (11), and BVSs II (19) and IV (13)

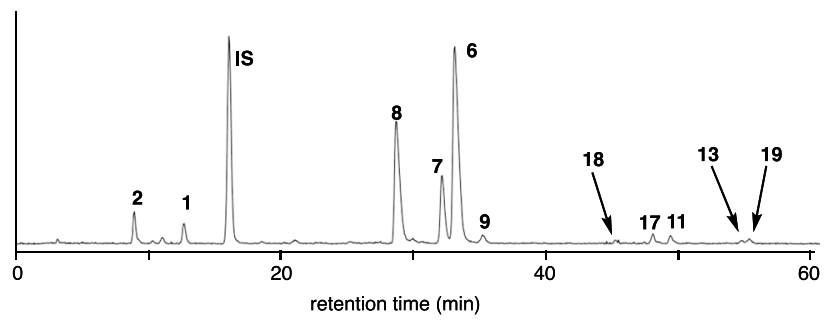

Fig. 4 Selected ion monitoring (SIM) chromatogram of a decoction of Achyranthes root

having four carboxylic acids, one of which is the C-28 of oleanolic acid, showed longer retention times.

\section{Saponin patterns of Achyranthes root and the effect of different extraction and preparation conditions on the amounts of the saponins}

Achyranthes roots in the Japanese market showed variable saponin patterns [32]. In an LC-MS analysis by selective ion monitoring of 11 saponins $(\mathbf{1}, \mathbf{2}, \mathbf{6}-\mathbf{9}, \mathbf{1 1}, \mathbf{1 3}, \mathbf{1 7}-\mathbf{1 9})$, three patterns of saponin composition were observed for the water extracts: (1) the saponins with sugar moiety at C-28 [ASs B (6), C (7) and D (8)] were the major constituents (Fig. 5a), (2) the saponins without sugar moiety at C-28 [BVSs II (19) and IV (13)] were the major constituents (Fig. 5b), and 3) mixtures of these saponins (Fig. 5c) [32]. In these samples, the amounts of CSs IVa (1) and V (2) were very small or negligible. However, in some samples stored for a long period and their color changed to dark, CSs IVa (1) and V (2), and AS B (6) were the major constituents (Fig. 5d).

When hot water was used for the extraction, ASs B (6), C (7) and D (8) were detected even from the samples whose water extract did not contain these saponins (Fig. 6). This was attributed to inactivation of endogenous esterase which hydrolyze the ester linkage at C-28 [32].

Fuchino et al. [36] investigated the effect of drying temperature of the root on the saponin constituents by LC-NMR/ MS. The pattern of the saponin constituents varied depending on the drying temperature. Chikusetsusaponins were the major constituents of the samples dried above $70{ }^{\circ} \mathrm{C}$, and from the roots dried at $100{ }^{\circ} \mathrm{C}$ for 3 days, they identified CSs IVa (1) and V (2), 28-desglucosyl CS V (4), and oleanolic acid 3-O-glucuronide (=momordin $\mathrm{Ib}, \mathbf{1 6}$ ). On the contrary, achyranthosides were detected from the roots dried below $50{ }^{\circ} \mathrm{C}$ [36]. Ultra-performance liquid chromatography coupled with quadrupole time-of-fright MS/MS (UPLC-QTOF-MS/MS) analysis was used for monitoring the effect of sulfur-fumigation of Achyranthes root [37]. The amounts of BVSs II (19), III (= AS C, 7) and IV (= AS G, 


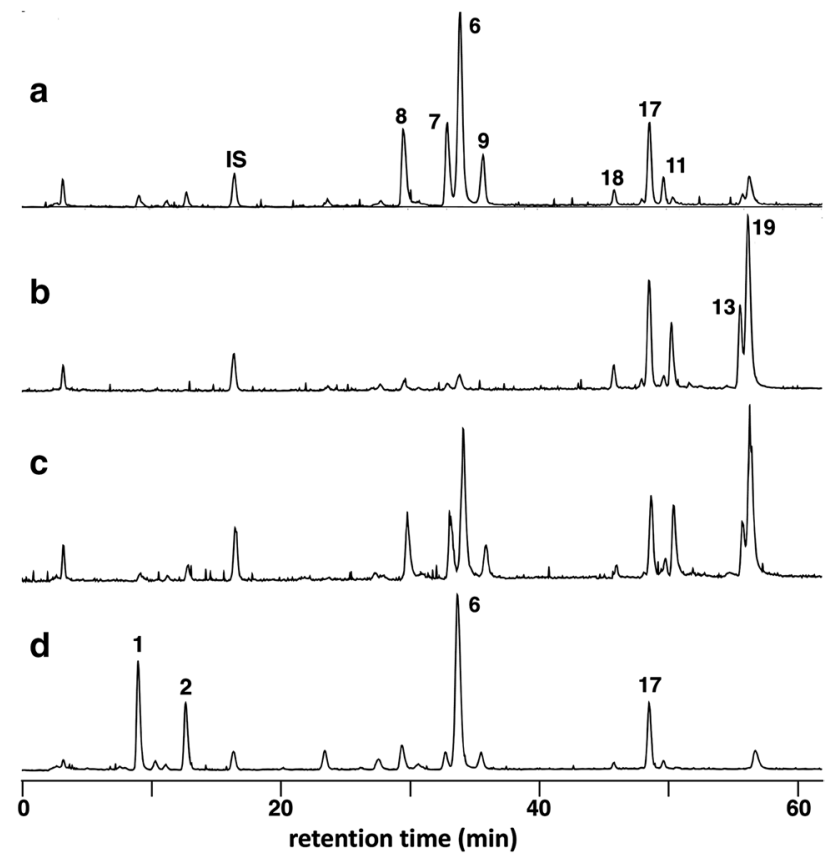

Fig. 5 Representative saponin patterns of Achyranthes root in the Japanese market. a Saponins with glucose moiety at C-28 are the major constituents; $\mathbf{b}$ saponins without glucose moiety at C-28 are the major constituents; $\mathbf{c}$ a mixed pattern of $\mathbf{a}$ and $\mathbf{b}$; $\mathbf{d}$ a pattern of samples after long storage

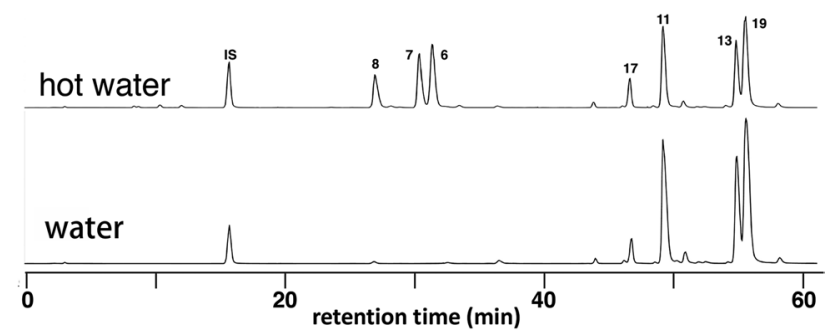

Fig. 6 Comparison of the water and hot water extracts of the same crude drug

11) decreased to $28 \%, 38 \%$ and $37 \%$ of the original amounts, respectively, after $2 \mathrm{~h}$ of heavy sulfur-fumigation (the weight ratio of sulfur to herbal material $=1: 20$ ).

The amounts of the saponins in the extract were variable depending on the extraction conditions. The amounts of the saponins prepared by three extraction methods: water extraction for $24 \mathrm{~h}$, decoction for $30 \mathrm{~min}$, and reflux for $3 \mathrm{~h}$, of the same crude drug sample were compared [25]. AS B (6) and D (8) were the major saponins and the amounts of CSs IVa (1) and V (2) were negligible in the water extract. The amounts of AS B (6), C (7) and D (8), especially that of AS B (6), increased and small amounts of CS IVa (1) and $\mathrm{V}(\mathbf{2})$ were detected in the decoction. When the sample was extracted under reflux, the amounts of CS IVa (1) and
V (2) increased greatly and that of AS B (6) also increased, whereas the amounts of the other saponins were similar to those in the decoction. The results suggested that CS IVa (1) and V (2) were mainly formed from AS C (7) and D (8), respectively. On the other hand, the relative amount of AS B (6) among achyranthosides increased on prolonged heating, because the dicarboxylic acid moiety of AS B (6) forms a six-membered ring structure and more stable compared to the dicarboxylic acid moiety of the other achyranthosides. The amounts of BVS II (19) and IV (13) in decoction were largely decreased compared with those in the water extract prepared from the same sample. As large amounts of these saponins were found in the precipitates formed by heating of the water extract, these saponins were seemed to precipitate out under heating [32]. When saponins were extracted with $\mathrm{BuOH}$ from the water extract, the relative amounts of highly polar saponins, AS D (8) and SASs B (17) and D (18), in the $\mathrm{BuOH}$ extract decreased. In addition, when reagent grade $\mathrm{BuOH}$ was used, oxidative decarboxylation of AS B (6) and BVS II (19) occurred resulting in formation of AS E (9) and SS D (20), respectively. As these changes were not observed with HPLC grade $\mathrm{BuOH}$, which contains not more than $5 \mathrm{ppm}$ of peroxide impurities, the change was attributable to the peroxide impurities contained in the reagent grade $\mathrm{BuOH}$ [32].

Localization of the saponin constituents were examined by Jaiswal et al. They separated root samples to cortex, medullary rays and tertiary vascular bundles and analyzed each part to show that, although saponins were detected in all the parts, the amount was highest in cortex followed by medullary rays and tertiary vascular bundles [38]. The contents of oleanolic acid in the acid hydrolysate of the root, stem and leaf of A. bidentata were also investigated [39]. All the three parts contained oleanolic acid, and the contents (\%) were the highest in early August (root, $c a .8 \%$; stem and leaf, $c a .4 \%$ ). In early September, the amount in the root dropped dramatically (ca. 1\%), whereas the decrease in the stem and leaf was small (ca. 3\%). In October and November, the content was almost constant in the root (ca.3\%) and the stem (ca.1\%).

\section{Biological activity of the saponins of Achyranthes root}

Oleanane saponins are one of the major constituents of Achyranthes root and some biological activities have been reported for the saponins. Yoshikawa et al. [11] reported hypoglycemic effect of BVSs II (15), III (= AC C, 7) and IV (13). A fraction composed of ASs E (9) and F (10) prepared from Achyranthes root potently inhibited the interaction between polymorphonuclear leukocytes (PMNs) and E-selectin [9]. AS H (12) methyl ester showed strong antiproliferative effect against human breast cancer cells with 
induction of apoptosis, whereas the effect of methyl esters of ASs C (7) and E (9) was moderate [40]. A saponin fraction prepared from the $\mathrm{BuOH}$ soluble fraction of the $70 \%$ EtOH extract of Achyranthes root suppressed IL-1 $\beta$ induced apoptosis and nuclear factor $\mathrm{\kappa B}$ activation in rat chondrocytes [41]. Methyl and butyl esters of oleanolic acid saponins including ASs A (5), C (7), D (8), and E (9), and CSs IVa (1) and V (2) isolated from the $\mathrm{BuOH}$ soluble fraction of the $\mathrm{MeOH}$ extract of A. bidentata were reported to inhibit the formation of osteoclast-like multinucleated cells induced by $1 \alpha, 25$-dihydroxyvitamin $\mathrm{D}_{3}$ [23]. Saponins of A. bidentata were also reported to promote osteogenic differentiation of bone marrow stromal cells through the ERK MAPK signaling pathway [42]. Ginsenoside Ro $(=\mathrm{CS} \mathrm{V}, 2)$ inhibited adhesion, migration and invasion of colon cancer cells HT29 [43]. The cytotoxicity of $A$. fauriei roots increased by heat processing and the cytotoxic principle against human hepatoma SK-Hep-1 cells was identified as CS IVa (1) [44]. The amounts of these compounds also increased in salt-processed Achyranthes root and contributed to the protective effect against LPSinduced acute kidney injury [45].

\section{Conclusion}

Triterpene saponins are one of the major constituents of Achyranthes root. Several groups isolated characteristic saponins with a dicarboxylic acid group and, in some cases, different names were given to one compound. This review sorted out all the triterpene saponins with dicarboxylic acid moiety isolated from Achyranthes root and clarified their relationships. Although the dicarboxylic acid moiety of the saponins has been postulated to be formed by oxidative cleavage of a terminal monosaccharide unit, actual precursor and reactions involved in the biosynthesis remain to be investigated. In recent years, more attention has been paid to the high molecular weight constituents of Achyranthes root in relation to the biological activities. For example, effects of the polypeptides on nervous system $[46,47]$ and effects of the polysaccharides on bone metabolism [48, 49] have been reported. Nevertheless, saponins are one of the representative constituents of Achyranthes root, and although some biological activities have been reported for the saponins, further investigations on the saponins will be necessary to understand the medicinal property of Achyranthes root and use the crude drug effectively.

Supplementary Information The online version contains supplementary material available at https://doi.org/10.1007/s11418-021-01591-1.
Open Access This article is licensed under a Creative Commons Attribution 4.0 International License, which permits use, sharing, adaptation, distribution and reproduction in any medium or format, as long as you give appropriate credit to the original author(s) and the source, provide a link to the Creative Commons licence, and indicate if changes were made. The images or other third party material in this article are included in the article's Creative Commons licence, unless indicated otherwise in a credit line to the material. If material is not included in the article's Creative Commons licence and your intended use is not permitted by statutory regulation or exceeds the permitted use, you will need to obtain permission directly from the copyright holder. To view a copy of this licence, visit http://creativecommons.org/licenses/by/4.0/.

\section{References}

1. Ministry of Health, Labor and Welfare (2021) The Japanese Pharmacopoeia eighteenth Edition (Ministry Notification No. 220 of Jun. 7, 2021), p 1928

2. Royal Botanic Gardens Kew, Plants of the World Online. Achyranthes bidentata Blume. http://powo.science.kew.org/taxon/urn: lsid:ipni.org:names:58678-1. Accessed 12 Sept 2021

3. He X, Wang X, Fang J, Chang Y, Nina N, Guo H, Huang L, Huang $X$ (2017) The genus Achyranthes: a review on traditional uses, phytochemistry, and pharmacological activities. J Ethnopharmacol 203:260-278. https://doi.org/10.1016/j.jep.2017.03.035

4. Takemoto T, Ogawa S, Nishimoto N, Taniguchi S (1967) Studies on the constituents of Achyranthis Radix. IV. Isolation of the insectmoulting hormones from formosan Achyranthes spp. Yakugaku Zasshi 87:1478-1480. https://doi.org/10.1248/yakushi1947. $87.12 \_1478$

5. Zhang M, Zhou Z-Y, Wang J, Cao Y, Chen X-X, Zhang W-M, Lin L-D, Tan J-W (2012) Phytoecdysteroids from the roots of Achyranthes bidentata Blume. Molecules 17:3324-3332. https:// doi.org/10.3390/molecules17033324

6. Ida Y, Katsumata M, Satoh Y, Shoji J (1994) Glucuronide saponins of oleanolic acid from Achyranthes fauriei roots. Planta Med 60:286-287. https://doi.org/10.1055/s-2006-959481

7. Ida Y, Satoh Y, Katoh M, Katsumata M, Nagasao M, Yamaguchi K, Kamei H, Shoji J (1994) Achyranthosides A and B, novel cytotoxic saponins from Achyranthes fauriei root. Tetrahedron Lett 35:6887-6890. https://doi.org/10.1016/0040-4039(94)85032-1

8. Ida Y, Satoh Y, Katsumata M, Nagasao M, Shoji J (1995) Achyranthosides $\mathrm{C}$ and $\mathrm{D}$, novel glucuronide saponins from Achyranthes fauriei root. Chem Pharm Bull 43:896-898. https://doi.org/ 10.1248/cpb.43.896

9. Ida Y, Satoh Y, Katsumata M, Nagasao M, Hirai Y, Kajimoto T, Katada N, Yasuda M, Yamamoto T (1998) Tow novel oleanolic acid saponins having a sialyl Lewis $\mathrm{X}$ mimetic structure from Achyranthes fauriei root. Bioorg Med Chem Lett 8:2555-2558. https://doi.org/10.1016/S0960-894X(98)00457-0

10. Ando H, Fukumura M, Hori Y, Hirai Y, Toriizuka K, Kuchino Y, Ida Y (2008) Tow new glucuronide saponins, achyranthosdes G and $\mathrm{H}$, from Achyranthes fauriei root. J Nat Med 62:57-62. https:// doi.org/10.1007/s11418-007-0183-2

11. Yoshikawa M, Murakami T, Kadoya M, Matsuda H, Yamahara J, Muraoka O, Murakami N (1995) Betavulgarosides I, II, III, IV, and $\mathrm{V}$, hypoglycemic glucuronide saponins from the roots and leaves of Beta vulgaris L. (sugar beet). Heterocycles 41:16211626. https://doi.org/10.3987/COM-95-7111

12. Yoshikawa M, Murakami T, Kadoya M, Matsuda H, Muraoka O, Yamahara J, Murakami N (1996) Medicinal foodstuffs. III. 
Sugar beet. (1): Hypoglycemic oleanolic acid oligoglycosides, betavulgarosides I, II, III, and IV, from the root of Beta vulgaris L. (Chenopodiaceae). Chem Pharm Bull 44:1212-1217. https:// doi.org/10.1248/cpb.44.1212

13. Yoshikawa M, Murakami T, Kadoya M, Yamahara J, Matsuda $\mathrm{H}$ (1998) Medicinal foodstuffs. XV. Sugar beet. (2): structures of betavulgarosides V, VI, VII, VIII, IX, and X from the roots and leaves of sugar beet (Beta vulgaris L., Chenopodiaceae). Chem Pharm Bull 46:1758-1763. https://doi.org/10.1248/cpb.46.1758

14. Yoshikawa M, Murakami T, Hirano K, Matsuda H, Yamahara J, Ohtani K, Kasai R, Yamasaki K (1998) Absolute stereo structures of spinacosides $\mathrm{C}$ and $\mathrm{D}$ with a novel acetal type substituent from Spinacia oleracea (spinach) and Basella rubra (Indian spinach). Heterocycles 49:93-96. https://doi.org/10.3987/COM-98-S41

15. Wang G-S, Zhou X-P, Yang X-H, Xu J-D (2004) Study on the acidic triterpenoid saponins of Achyranthes bidentata Bl. Chin J Med Chem 14:40-42

16. Wang G-S, Cong D-L, Yang J-Z, Yang X-H (2005) The triterpenoid saponins in Achyranthes bidentata Bl. Chin J Med Chem $15: 224-226$

17. Mitaine-Offer A-C, Marouf A, Pizza C, Khanh TC, Chauffert B, Lacaille-Dubois M-A (2001) Bidentatoside I, a new triterpene saponin from Achyranthes bidentata. J Nat Prod 64:243-245. https://doi.org/10.1021/np000464a

18. Mitaine-Offer A-C, Marouf A, Hanquet B, Birlirakis N, Lacaille-Dubois M-A (2001) Two triterpene saponins from Achyranthes bidentata. Chem Pharm Bull 49:1492-1494. https:// doi.org/10.1248/cpb.49.1492

19. Marouf A, Desbene S, Khanh TC, Wagner H, Correia M, Chauffert B, Lacaille-Dubois MA (2001) Triterpene saponins from the roots of Achyranthes bidentata. Pharm Biol 39:263267. https://doi.org/10.1076/phbi.39.4.263.5908

20. Jia S-P, Yu Z-Y, Hao Z-F, Li J-X (2006) Isolation and identification of triterpenoids from root of Achyranthes bidentata in Henan, China. J Chin Mater Med 31:1244-1247

21. Qi N-X, Jia S-P, Hao Z-F, Li J-X (2005) Isolation and identification of the chemical constituents of radix from Achyranthes bidentata. Chin J Med Chem 15:162-166

22. Wei H-L, Li Y-J, Chen J, Li P (2012) Triterpenoid saponins in roots of Achyranthes bidentata. Chin J Nat Med 10:98-101. https://doi.org/10.3724/SP.J.1009.2012.00098

23. Li J-X, Hareyama T, Tezuka Y, Zhang Y, Miyahara T, Kadota $S$ (2005) Five new oleanolic acid glycosides from Achyranthes bidentata with inhibitory activity on osteoclast formation. Planta Med 71:673-679. https://doi.org/10.1011/s-2005-871275

24. Hoshino T, Narukawa Y, Haishima Y, Goda Y, Kiuchi F (2012) Two new sulfated saponins from Achyranthes root. J Nat Med 67:386-389. https://doi.org/10.1007/s11418-012-0684-5

25. Kawahara Y, Hoshino T, Morimoto H, Shimizu T, Narukawa Y, Fuchino H, Kawahara N, Kiuchi F (2016) LC-MS-based quantification method for Achyranthes root saponins. J Nat Med 70:102-106. https://doi.org/10.1007/s11418-015-0942-4

26. Murakami T, Hirano K, Yoshikawa M (2001) Medicinal foodstuffs. XXIII. Structures of new oleanane-type triterpene oligoglycosides, basellasaponins A, B, C, and D, from the fresh aerial parts of Basella rubra L. Chem Pharm Bull 49:776-779. https://doi.org/10.1248/cpb.49.776

27. Lavaud C, Beauviére S, Massiot G, Men-Olivier LL, Bourdy G (1996) Saponins from Pisonia umbellifera. Phytochemistry 43:189-194. https://doi.org/10.1016/0031-9422(96)00253-1

28. Iwamoto M, Okabe H, Yamauchi T (1985) Studies on the constituents of Momordica cochinchinensis Spreng. II. Isolation and characterization of the root saponins, momordins I, II and III. Chem Pharm Bull 33:1-7. https://doi.org/10.1248/cpb.33.1

29. Yoshikawa M, Murakami T, Inaduki M, Hirano K, Yamahara J, Masuda H (1997) Absolute stereostructures of betavulgarosides
III and IV, inhibitors of glucose absorption, from the roots of Beta vulgaris L. (sugar beet). Chem Pharm Bull 45:561-563. https://doi.org/10.1248/cpb.45.561

30. Murakami T, Matsuda H, Inadzuki M, Hirano K, Yoshikawa M (1999) Medicinal Foodstuffs. XVI. Sugar beet. (3): Absolute stereostructures of betavulgarosides II and IV, hypoglycemic saponins having a unique substituent, from the roots of Beta vulgaris L. Chem Pharm Bull 47:1717-1724. https://doi.org/ 10.1248/cpb.47.1717

31. Zhu S, Li Y, Yu B (2008) Synthesis of betavulgaroside II, a representative triterpene seco-glycoside. J Org Chem 73:49784985. https://doi.org/10.1021/jo800669h

32. Kuwada K, Kawase S, Nakata K, Shinya N, Narukawa Y, Fuchino H, Kawahara N, Kiuchi F (2020) LC-MS analysis of saponins of Achyranthes root in the Japanese Market. J Nat Med 74:135-141. https://doi.org/10.1007/s11418-019-01355-y

33. Li J, Qi H, Qi L-W, Yi L, Li P (2007) Simultaneous determination of main phytoecdysones and triterpenoids in Radix Achyranthes bidentatae by high-performance liquid chromatography with diode array-evaporative light scattering detectors and mass spectrometry. Anal Chim Acta 596:264-272. https://doi.org/10.1016/j.aca. 2007.05.016

34. Li J, Li P, Li H-J, Song Y, Bi Z-M, Li Y-J (2007) Simultaneous quantification and qualification of eight triterpenoids in Radix Achyranthis Bidentate by high-performance liquid chromatography with evaporative light scattering detection and mass spectrometric detection. J Sep Sci 30:843-850. https://doi.org/10.1002/ jssc. 200600341

35. Li Y-J, Wei H-L, Qi L-W, Chen J, Ren M-T, Li P (2010) Characterization and identification of saponins in Achyranthes bidentata by rapid-resolution liquid chromatography with electrospray ionization quadrupole time-of-flight tandem mass spectrometry. Rapid Commun Mass Spectrom 24:2975-2985. https://doi.org/10.1002/ rcm. 4728

36. Fuchino H, Hishida A, Akagi K, Kiuchi F, Kawahara N (2012) Quality evaluation of crude drugs using LC-NMR/MS (1) in the cultivation and processing of Achyranthes roots. Shoyakugaku Zasshi 66:1-16

37. Kang C, Zhao D, Kang L, Wang S, Lv C, Zhou L, Jiang J-Y, Yang W, Li J, Huang L-Q, Guo L (2018) Elucidation of characteristic sulfur-fumigated markers and chemical transformation mechanism for quality control of Achyranthes bidentate Blume using metabolome and sulfur dioxide residue analysis. Front Plant Sci 9:790. https://doi.org/10.3389/fpls.2018.00790

38. Jaiswal Y, Liang Z, Ho A, Chen H, Williams L, Zhao Z (2018) Tissue-based metabolite profiling and quantitative comparison of two species of Achyranthes roots by use of UHPLC-QTOF MS and laser micro-dissection. J Pharm Anal 8:10-19. https://doi.org/ 10.1016/j.jpha.2017.06.006

39. Li J, Hu Z (2009) Accumulation and dynamic trends of triterpenoid saponin in vegetative organs of Achyranthus bidentata. J Integr Plant Biol 51:122-129. https://doi.org/10.1111/j.17447909.2008.00764.x

40. Fukumura M, Ando H, Hirai Y, Toriizuka K, Ida Y, Kuchino Y (2009) Achyranthoside H methyl ester, a novel oleanolic acid saponin derivative from Achyranthes fauriei roots, induces apoptosis in human breast cancer MCF-7 and MDA-MB-453 cells via a caspase activation pathway. J Nat Med 63:181-188. https://doi. org/10.1007/s11418-008-0311-7

41. Xu X-X, Zhang X-H, Diao Y, Huang Y-X (2017) Achyranthes bidentate saponins protect rat articular chondrocytes against nterleukin-1 $\beta$-induced inflammation and apoptosis in vitro. Kaohsiung J Med Sci 33:62-68. https://doi.org/10.1016/j.kjms.2016.11. 004

42. He G, Guo W, Lou Z, Zhang H (2014) Achyranthes bidentata saponins promote osteogenic differentiation of bone marrow stromal 
cells through the ERK MAPK signaling pathway. Cell Biochem Biophys 70:467-473. https://doi.org/10.1007/s12013-014-9942-3

43. Jiang Z, Qian J, Dong H, Yang J, Yu X, Chen J, Chen H, Shi Q, Jia L (2017) The traditional Chinese medicine Achyranthes bidentata and our de novo conception of its metastatic chemoprevention: from phytochemistry to pharmacology. Sci Rep 7:3888. https:// doi.org/10.1038/s41598-017-02054-y

44. Yoo HH, Kwon SW, Park JH (2006) The cytotoxic saponin from heat-processed Achyranthes fauriei roots. Biol Pharm Bull 29:1053-1055. https://doi.org/10.1248/bpb.29.1053

45. Wang S, Zeng M, Li B, Kan Y, Zhang B, Zheng X, Feng W (2020) Raw and salt-processed Achyranthes bidentata attenuate LPS-induced acute kidney injury by inhibiting ROS and apoptosis via an estrogen-like pathway. Biomed Pharmacother 129:110403. https://doi.org/10.1016/j.biopha.2020.110403

46. Peng S, Xu L, Ma J-Y, Gu X-S, Sun C (2018) Achyranthes bidentata polypeptide protects dopaminergic neurons from apoptosis induced by rotenone and 6-hydroxydopamine. Neural Regen Res 13:1981-1987. https://doi.org/10.4103/1673-5374.239446
47. Li M, Zhu Y, Tang L, Xu H, Zhong J, Peng W, Yuan Y, Gu X, Wang H (2021) Protective effects and molecular mechanisms of Achyranthes bidentata polypeptide k on Schwann cells. Ann Transl Med 9:381. https://doi.org/10.21037/atm-20-2900

48. Song D, Cao Z, Huang S, Tickner J, Li N, Qiu H, Chen X, Wang C, Chen K, Sun Y, Dong S, Xu J (2017) Achyranthes bidentata polysaccharide suppresses osteoclastogenesis and bone resorption via inhibiting RANKL signaling. J Cell Biochem 119:4826-4835. https://doi.org/10.1002/jcb.26682

49. Zhang S, Zhang Q, Zhang D, Wang C, Yan C (2018) Anti-osteoporosis activity of a novel Achyranthes bidentata polysaccharide via stimulating bone formation. Carbohydr Polym 184:288-298. https://doi.org/10.1016/j.carbpol.2017.12.070

Publisher's Note Springer Nature remains neutral with regard to jurisdictional claims in published maps and institutional affiliations. 